\title{
Discovering SOCIABLE: Using a Conceptual Model to Evaluate the Legibility and Effectiveness of Backchannel Cues in an Entertainment Scenario
}

\author{
Antonio Andriella ${ }^{1}$ Rubén Huertas-García ${ }^{2}$ Santiago Forgas-Coll ${ }^{2}$ Carme $_{\text {Torras }}{ }^{1}$ and Guillem Alenyà ${ }^{1}$
}

\begin{abstract}
Robots are expected to become part of everyday life. However, while there have been important breakthroughs during the recent decades in terms of technological advances, the ability of robots to interact with humans intuitively and effectively is still an open challenge.

In this paper, we aim to evaluate how humans interpret and leverage backchannel cues exhibited by a robot which interacts with them in an entertainment context. To do so, a conceptual model was designed to investigate the legibility and the effectiveness of a designed social cue, called SOCial ImmediAcy BackchanneL cuE (SOCIABLE), on participant's performance. In addition, user's attitude and cognitive capability were integrated into the model as an estimator of participants' motivation and ability to process the cue. In working toward such a goal, we conducted a two-day long user study $(\mathrm{N}=114)$ at an international event with untrained participants who were not aware of the social cue the robot was able to provide. The results showed that participants were able to perceive the social signal generated from SOCIABLE and thus, they benefited from it. Our findings provide some important insights for the design of effective and instantaneous backchannel cues and the methodology for evaluating them in social robots.
\end{abstract}

\section{INTRODUCTION}

Social Robotics aims at creating robots with the ability to exhibit social behaviours [1]. Social Robots have already been employed in several contexts, such as education [2], healthcare [3], [4], and entertainment [5].

A socially assistive robot is one that employs a set of interaction strategies, combining verbal and non-verbal communication channels, to tailor the degree of assistance to the particular user's need [6]. In order to effectively design motivational strategies and thus influence a human's behaviour as well as their attitude toward a task, two key capabilities are crucial: i) the robot's ability to communicate effectively and ii) its capability to impact on the human's decisions. To address the first aspect, several studies have investigated how verbal and non-verbal social cues can be integrated into a robotic system enabling humans to experience a more natural

*This work has been partially funded by the EU project SOCRATES H2020-MSCA-ITN-721619, by the European Union's Horizon 2020 under ERC Advanced Grant CLOTHILDE (no. 741930), by the Spanish Ministry of Science and Innovation HuMoUR TIN2017-90086-R, and by the State Research Agency through the María de Maeztu Seal of Excellence to IRI (MDM-2016-0656).

${ }^{1}$ A. Andriella, C. Torras, G. Alenyà are with Institut de Robòtica i Informàtica Industrial, CSIC-UPC, C/Llorens i Artigas 4-6, 08028 Barcelona, Spain. \{aandriella, torras, galenya\}@iri.upc.edu

${ }^{2} \mathrm{R}$. Huertas and S. Forgas are with the Faculty of Economics and Business, Universitat de Barcelona, Av Diagonal 690, 08034 Barcelona, Spain. $\{$ santiago.forgas, rhuertas\}@ub.edu

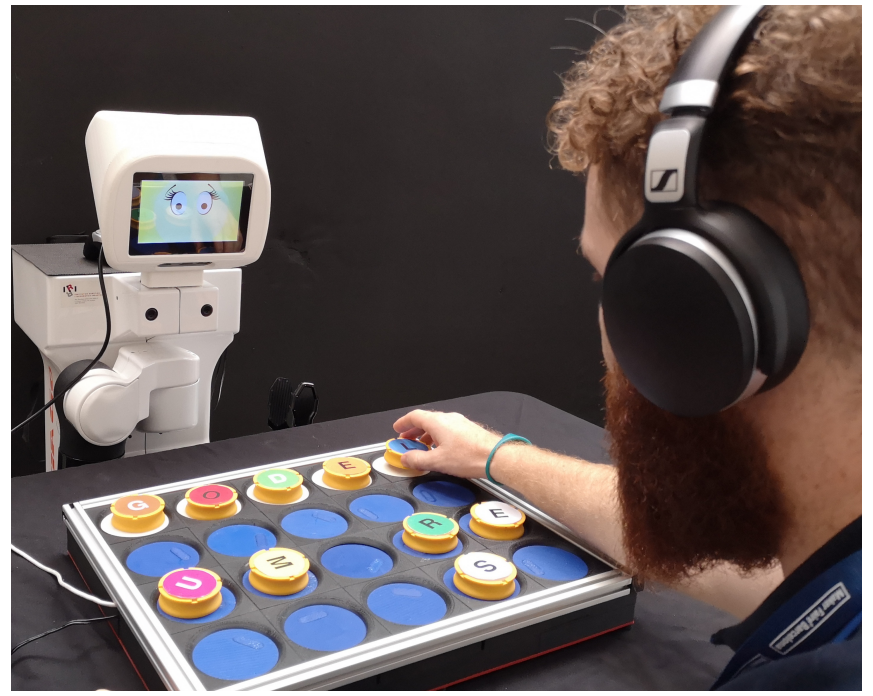

Fig. 1: Participant plays the puzzle game.

interaction [7]. Regarding the second aspect, an effort from the robotics community has been made to develop persuasive strategies to shape human attitudes as well as motivation strategies to easily accomplish a given objective [8].

Social intelligence enables robots to interact socially, and react accordingly as well as to communicate their internal state through verbal and non-verbal social cues [8]. Through a combination of social cues, a robot can convey what becomes interpreted as a social signal and thus influence the human counterpart [9]. For instance, when two people are interacting, one can nod his/her head (non-verbal cue) to convey understanding of what the other was saying (social signal). In the same way, a robot can leverage its social cues, such as arm movement and facial expression, in response to a human interlocutor's action. However, most of the current robotic systems still lack the ability to exhibit verbal and non-verbal cues in ways that are understandable and natural for humans [10].

While backchannels in linguistics refer to the way the listener responds to the speaker, here this concept defines SOCial ImmediAcy BackchanneL cuE (SOCIABLE). SOCIABLE is a kind of feedback that consists of a combination of verbal and non-verbal social cues, integrating non-lexical and phrasal backchannels with an instantaneous response to participant's actions while interacting with a robot in an entertainment context. SOCIABLE aims at effective 
persuasion, affecting the participant's behaviour and thus performance. Its design is the first contribution of this paper.

However, persuasive communication requires that the recipient be motivated to process the information and have sufficient ability to process it correctly [11]. Therefore, it is convenient to know the degree of motivation and cognitive abilities of recipients in order to have a clear interpretation of the effectiveness of the verbal and non-verbal stimuli emitted. As a consequence, in this study, we assess user's attitude and reasoning system (intuitive vs analytical thinking) as estimators of the degree of motivation and cognitive capacity respectively, and also as moderating variables of the effectiveness of communication.

Practical assessment and objective evaluation are always problematic in social robotics. The second contribution is the design of a conceptual model with the purpose of evaluating the effectiveness of SOCIABLE on the participants' performance, as well as investigating its relationship with participants' attitude and reasoning system. Furthermore, we evaluated whether and to what extent SOCIABLE was perceived by the participants as a clue and subsequently employed. In the proposed conceptual model, participants' reaction time and SOCIABLE act as antecedents of the number of mistakes, but at the same time, SOCIABLE plays a mediating role. Furthermore, participants' reasoning system and attitude are considered as moderating variables of the performance of the participant interacting with the robot.

To achieve this goal, we conducted a user study $(\mathrm{N}=114)$ in which untrained participants were asked to play a puzzle game with the collaboration of the robot (see Fig. 1). The robotic system built upon [12] was able to offer encouragement and clues at each participant's turn. Furthermore, it provided SOCIABLE, to discourage a participant when the lifted token was the wrong one. While participants were aware of the assistance the robot could offer them, they were not informed about SOCIABLE. From this study, we measured participants' reaction time, number of mistakes and whether they were able to grasp and understand the robot's feedback (SOCIABLE). Furthermore, we assessed participants' attitude and reasoning with a questionnaire and a test, respectively. The user study to evaluate the legibility and the effectiveness of SOCIABLE is the third contribution of this paper.

The results of our user study provided important insights for the design of effective and instantaneous backchannel cues. Finally, our findings revealed the importance of using a conceptual model that enables the inclusion of social constructs in the evaluation of social cues in human-robot interaction (HRI).

\section{RESEARCH QUESTIONS}

In this study, we aim to investigate the following research questions:

RQ1: (legibility) Will participants' reaction time as well as their attitude and reasoning system affect the detection of SOCIABLE?
RQ2: (effectiveness) When perceived, will SOCIABLE impact on the participants' behaviour and consequently on their performance during the game?

\section{RELATED WORK}

\section{A. The Importance of Social Cues in Social Robotics}

The importance of designing and developing robot's social cues is a key aspect for providing a more natural user's experience that has some important benefits: it can increase trust and acceptance and the user's overall engagement [13]. A lot of work has been done to investigate whether and to what extent verbal and non-verbal social cues can be deployed in a robot.

Anzalone et al. [13] proposed a methodology to evaluate user engagement based on metrics extracted by analysing non-verbal cues through non-invasive sensors. These metrics were employed by the robot as feedback to estimate the perceived social intelligence by the human and at the same time to quantify the number of changes elicited in the user. Kennedy et al. [14] explored the concept of nonverbal immediacy, to assess how it can improve robot's level of sociality. Furthermore, they evaluated how nonverbal immediacy can affect the participants' behaviour in a learning scenario. Similarly, as in [13], our metrics evaluate the influence of the provided signal and as [14] we aim to evaluate whether our designed social feedback can influence participants' behaviour. However, differently from [13], [14], our designed social feedback is a combination of verbal and non-verbal social cues. Jost et al. [15] explored the effectiveness of non-verbal and verbal robot's behaviour for increasing user's acceptance and trust. Chidambaram et al. [16] explored how manipulating body cues, gestures, and vocal cues could affect the persuasiveness of a humanlike robot. Ham et al. [17] investigated whether and to what extent gaze and gestures was better as social cues, as part of persuasion strategy, than gestures alone. Results reported that combining both the social cues increased the robot's persuasiveness. As [15], [16], and [17], we envisage that social cues play a key role in improving people's motivation. Nonetheless, in our study, we are interested in evaluating whether or not the designed social feedback can be recognised by participants with respect to their reaction time along with their reasoning system and degrees of motivation.

\section{B. The Importance of Social Signal Processing in Social Robotics}

The field of Social Signal Processing studies how to furnish artificial agents with social intelligence [18]. A common focus has been on investigating how robotic social cues can influence the perception of social signals and how this can improve the design of more socially intelligent robots [19]. Combining verbal and non-verbal social cues, a robot [20] or a conversational agent [21] can communicate what becomes interpreted as a social signal representing its internal state. Wiltshire et al. [22] and Fiore et al. [9] investigated the role of proxemic behaviour and gaze in a hallway navigation scenario. In particular, they focused 
on the effects the robot's social cues had on the types of social signals the participants reported after the interaction. In the same direction, Wiltshire et al. [22], evaluated how the same social cues, proxemic behaviour and gaze, could influence the interpersonal attributions made toward the robot and the estimation of its behaviour. This work was extended by Warta et al. [20], who examined the effect of different types of display and proxemic behaviours over repeated interactions on the participant's perception of the robot's social presence. Jerčić et al. [23] studied whether participants playing a serious game by collaborating with a robot, perceive the latter as a social agent based on a small subset of social cues. The work presented by Lobato et al. [24], examined the relationship between social cues and social signals. Specifically, they evaluated which social signals were reported by participants for a given set of social cues in a simulated surveillance task.

Our investigation is motivated by these studies. Here, we extended the robotic architecture presented in [12] designing specific social feedback to evaluate whether and to what extent the produced social signal affects the users' performance.

\section{The Importance of User's Attitude and Reasoning System in Social Robotics}

In most studies on HRI, users are considered as a homogeneous group that makes their decisions rationally and, consequently, without motivation or processability problems ([25]). However, for a long time, the literature on consumer behaviour has pointed to the opposite. That is, it considered consumers as a heterogeneous group, with different degrees of motivation in making decisions [11] and, in many decisions, they stated different abilities when taking mental shortcuts or using very simple criteria [26]. Although it is obvious that without motivation and using simple criteria it is impossible to make good decisions, it is feasible to achieve acceptable decisions taking into account the effort made and the benefit obtained [27]. Therefore, to better understand HRI it is important to consider users' classification criteria that allow more homogeneous groups to be formed based on their motivations and behaviours.

One of these classification criteria is attitude. In social psychology, the attitude is a psychological construct that aims to reflect a degree of affect (positive or negative) towards an object (a robot) resulting from information received, related to the object and previous diagnoses [28]. Although attitude is not observable, in market research it is made operational by combining stated beliefs about the object and degrees of importance assigned to them [29]. Beliefs reflect opinions formed over time, as a result of information received from multiple sources and stored in memory. However, the degree of importance reflects the relative weight of each of these beliefs in the formation of a positive or negative attitude toward the object. That is, human behaviour does not respond to the subconscious and inconstant forces that would make it impossible to establish patterns of behaviour, but, within the paradigms of social psychology, follows a certain logic. Since the attitude is based on information stored in the memory resulting from external information, the experience with similar or equal objects further determines the intention and, subsequently, the behaviour [28].

Among the operational elements that shape the attitude, the degree of importance is very similar to involvement [29]. Although this relationship between attitude and involvement was established decades ago [30], it has become an essential topic in market research. Since for any product it is possible to classify users by their degree of involvement, it is expected that the most involved, employ a more intense cognitive process in understanding the most relevant features and advantages of the product, while the least involved dedicate less time and effort [11]. In our context, we believe that any variable linked to involvement, such as attitude, can contribute to understanding the effectiveness of the robot's assistance in shaping the participants' behaviour. Hence, we adopted attitude as a moderating variable [30].

Another important variable to classify users who interact with robots is the reasoning system. According to the dualprocess theory [31], individuals use two different cognitive systems to gather and process information, and make decisions: a primitive system, similar to that of many advanced animals such as large apes, and another exclusive to humans, far superior to that of animals [31]. The first called System 1 is more ancient, autonomous and intuitive and does not require the use of memory, and the second called System 2, is more reflexive, analytical and deliberative, which requires the use of memory [32]. In general, individuals are more inclined to use System 1, which has led to qualifying humans as miserly cognitive [32]. Some evolutionary psychologists have tried to explain this bias, proposing that humans follow the same principle of energy efficiency as other living beings. And, since analytical reasoning consumes important energy resources, individuals try to avoid it replacing it, whenever possible, with fast and economic heuristics [33]. However, this does not mean making incorrect decisions, since many of these heuristics have been forming for thousands of years, as a result of overcoming survival challenges. And, in many situations, fast heuristics can generate better decisions than those of more complex processes [34]. Moreover, its consistency has also been demonstrated, that is, once a decision has been made through heuristics, it is rarely modified later [35] and then they are more efficient in routine than in stochastic environments, where System 2 responses are usually more effective [36]. This users' characteristic, as far as we know, had never been used in social robotics, but in some studies of new technologies [35]. In conclusion, we believe that this variable can play a key role as moderator in HRIs.

\section{SOCIAL IMMEDIACY BACKCHANNEL CUE}

Aiming to foster HRI and to establish a more natural and effective communication between them, we defined SOCIABLE. SOCIABLE was designed keeping in mind two main aspects that define social intelligence:

- comprehensible and intuitive, as it needs to be grasped and interpreted by humans without any effort, 


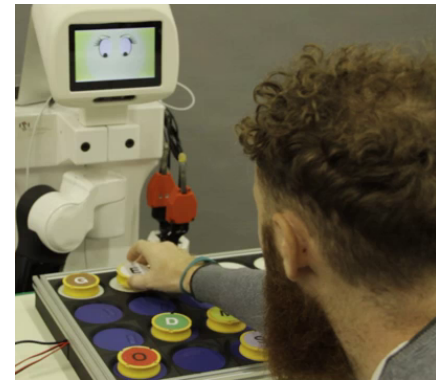

(a)

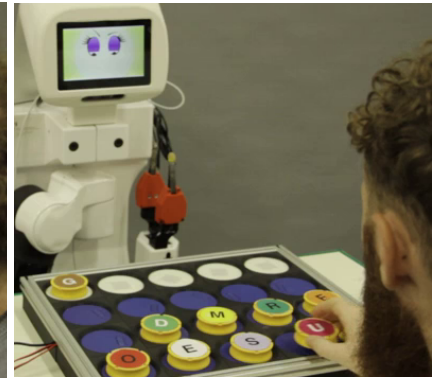

(b)
Fig. 2: Participant plays the puzzle game, whose solution is GODEL. At that state of the game, the participant is expected to pick token 'O'. In (a) the participant does not grasp SOCIABLE and places the token in the wrong location. In (b) the participant perceives SOCIABLE (thanks to its verbal and non-verbal backchannel cues) and moves the token back to its original location.

- immediate, as it needs to be instantaneous since the time of intervention is crucial in communication between two interlocutors.

Therefore, SOCIABLE combined verbal (voice) and nonverbal (facial expression) backchannel cues at a specific time during the game: immediately after a participant lifted a token. Leveraging SOCIABLE, the robot may influence the participants' actions; namely, it may discourage them from placing the token in a wrong location and encourage them to move it back to its original location (see Fig. 2).

Concerning the non-verbal cues, the facial expressions were designed according to the findings reported by Kalegina et al. in [37] and evaluated in a pilot study with 60 participants. Thus, the facial expressions related to SOCIABLE were: happy if the chosen token was the correct one, and sad, confused or angry if it was the wrong one. The robot switched from sad to confused and angry depending on the number of repeated unsuccessful attempts (see Fig. 3).

Regarding the verbal cues, very short sentences were used to communicate to the participant the outcome of their action based on the concepts of non-lexical and phrasal backchannels in linguistics. The robot said words like: "Great!", "Yeah!", "Wow!" when the participant picked the correct token. On the other hand, when the participant grasped the wrong token, the robot says "Mmmh", "Really?", "Are you sure?". Finally, we were able to provide SOCIABLE, with a delay respect to the time the user picked a token of less than $0.5 \mathrm{sec}$.

\section{CONCEPTUAL MODEL}

In studies on consumer behaviour, it is considered that the purchase of a product is not something random or subconscious, but rather the opposite, which obeys relationship patterns that are reflected in conceptual models, supported by theory. The following study has not been proposed as an experiment, but rather as a procedure to evaluate a working method in order to improve it. To this end, we propose

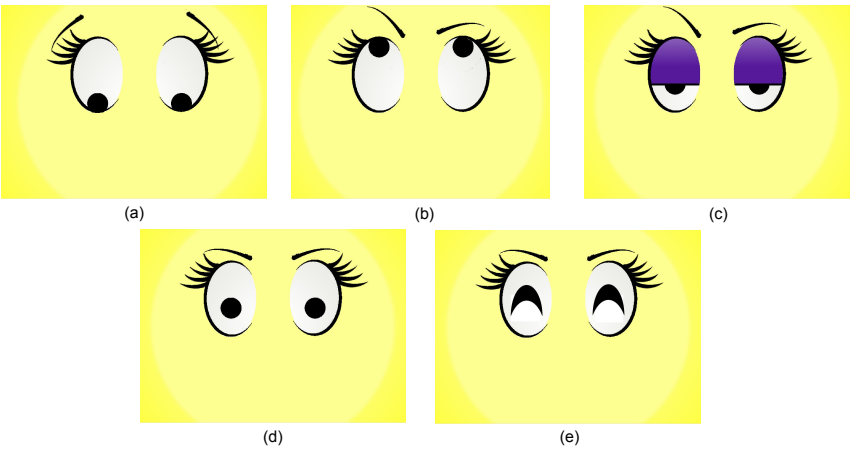

Fig. 3: Robot's facial expressions: (a) sad, (b) confused, (c) angry, (d) neutral, and (e) happy.

a conceptual model inspired by the study of methodstime measurement to analyse a task [38]. This procedure requires describing the system in detail, determining its operations, establishing sequential diagram and identifying possible sources of error and human reliability [39].

We observe that mistakes made when performing a task are due to multiple causes, including the time spent and the intervention of another agent, who can also mediate (in Fig. 4, the blue arrows reflect this relationship). A mediator variable $\mathrm{M}$ explains the relationship between an independent variable $\mathrm{X}$ and its dependent variable Y. According to [38], we define a base model (see Fig. 4, blue rectangle) in which participants' reaction time and SOCIABLE act as antecedents of the number of mistakes, moreover SOCIABLE acts as a mediator between participants' reaction time and number of mistakes. Finally, with respect to the base model, we aim to evaluate the following hypotheses:

H1. In HRI in an entertainment scenario, the user's reaction time is positively related to SOCIABLE.

H2. In HRI in an entertainment scenario, the user's reaction time is positively related to the number of mistakes.

H9. In HRI in an entertainment scenario, SOCIABLE, when perceived by a user, is positively related to the number of mistakes.

H10. SOCIABLE when perceived by a user is a mediator between user's reaction time and the number of mistakes.

Furthermore, the number of mistakes as well as SOCIABLE can be moderated by the degree of involvement and the cognitive abilities of the subject (in Fig. 4, the orange arrows reflect this relationship). A moderator variable $\mathrm{W}$ is a variable that affects the strength of the relation between an independent variable $\mathrm{X}$ and a dependent variable $\mathrm{Y}$. With respect to the extended model, we aim to evaluate the following hypotheses:

H3. User's reasoning system moderates the relationship between the user's reaction time and SOCIABLE when perceived by the user.

H4. User's reasoning system moderates the relationship between the user's reaction time and the number of mistakes. 


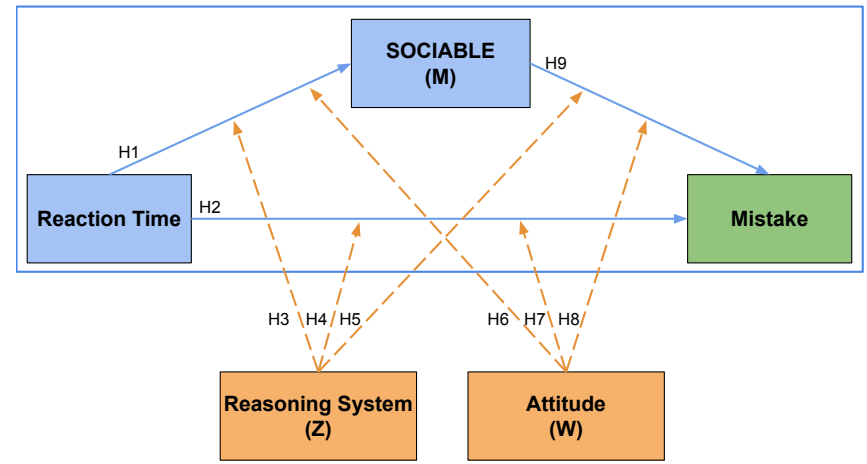

Fig. 4: Conceptual model which illustrates the relationship between the variables. The blue rectangle highlights the base model in which SOCIABLE (M) has a mediator role between Reaction Time and Mistake. The extended model includes in the base model, Reasoning System (Z) and Attitude (W) that have a moderator role.

H5. User's reasoning system moderates the relationship between SOCIABLE, when perceived by the user, and the number of mistakes.

H6. User's attitude moderates the relationship between the user's reaction time and SOCIABLE when perceived by the user.

H7. User's attitude moderates the relationship between the user's reaction time and the number of mistakes.

H8. User's attitude moderates the relationship between SOCIABLE, when perceived by a user, and the number of mistakes.

\section{FIELD WORK}

\section{A. The Puzzle Game}

In the proposed scenario, the participants played a game with the assistance of a robot. It consisted of composing the name of a Nobel Prize Winner with the tokens available on the board, trying to minimise the number of mistakes. With the purpose of fostering HRIs, we designed a puzzle game whose complexity was defined by the numbers of tokens available on the board; only 5 were needed to compose the word and there was only one correct solution.

\section{B. Apparatus}

In the study we used an electronic board and a TiaGo robot as shown in Fig. 1. The robotic platform and its architecture has already been presented in our previous work [12] where, in addition to voice communication, we used gestures (the robot arm moving) to provide assistance to the user. We concluded that this non-verbal communication channel was slow and not very effective. Here, on the contrary, we provide effective interactions using facial expressions as non-verbal backchannel communication. Consequently, the robot could furnish assistance through two communication channels: voice and facial cues.

Regarding the degrees of assistance, the robot could encourage the user or give a clue (look to your left, or to your right). Selecting one action or another was randomised, guaranteeing that all the participants received the same percentage of aids. The assistance was provided at each user's turn, that is, before a participant moved a token. Additionally the robot provided SOCIABLE, as already mentioned in Sec. IV, immediately after a user grasped a token.

With the purpose of detecting the location of each token, we used an electronic board as reported in [40]. Use of this technology allowed us to overcome the current limitations in uncontrolled environments that arise when perception is based on vision systems. As a result, we were able to detect not only when a token was placed in another location with $100 \%$ accuracy, but also when it was only lifted. This last capability enabled us to make SOCIABLE an instantaneous feedback.

\section{Procedure}

The study was conducted in a booth at the MakerFaire in Barcelona, following these steps:

1) Briefing $(5 \mathrm{~min})$ : The experimenter informed each participant of the procedure and asked their permission to gather their data for scientific purposes. Each participant was requested to fill out an informed consent form. Next, the experimenter explained the objective of the game without providing any clues on the kind of assistance the robot could furnish. In particular, as it was the aim of our study, none of the participants was informed of the capability of the robot to provide SOCIABLE. They were told to interact naturally with the robot while respecting the rules of the game. Furthermore, the participants were requested to try to complete the game with the lowest number of mistakes, regardless of the completion time.

2) Interaction with the robot $(5 \mathrm{~min})$ : Participants were asked to sit at a table with a board already set up to start the game (see Fig. 1). A TiaGo robot was placed opposite to them and they were told it would take care of providing information about the game. A headset with noise cancellation was provided to each participant in order to limit the sources of distraction and keep them focused on the task.

3) Questionnaires: Once the game was completed, each participant was asked to fill in a questionnaire. Questions had to be evaluated using a 5-point Likert scale ranging from 1 to 5 from completely disagree (1) to completely agree (5). 3 statements evaluated the attitude's construct as in [41]: i) I think it's a good idea to use the robot, ii) the robot would make life more interesting, iii) it's good to make use of the robot. The analysis of reliability for attitude returned Cronbach's $\alpha$ equals to 0.901 (CR=0.90 and $\mathrm{AVE}=0.78)$. Then, participants filled a Cognitive Reflection Test (CRT), which is a 7 questions test (three numerical and four non-numerical) with the characteristic of pointing toward a simple but incorrect answer, while the correct answer requires mental effort to find it [32], [42].

4) Measures: To assess our research questions, we define the following indicators:

- participant's reaction_time: the time the participant took to place a token after picking it and getting SOCIABLE 


\begin{tabular}{c|l|lll}
\hline Hypothesis & Antecedent & Coeff. & SE & p \\
\hline H1 & Reaction Time (RT) & 0.24 & 0.008 & 0.006 \\
& SOCIABLE (SF) & - & - & - \\
H3 & Reasoning System (RE) & -2.46 & 1.76 & 0.16 \\
& RTxRE & 0.71 & 0.34 & 0.03 \\
& SFxRE & - & - & - \\
H6 & Attitude (AT) & 0.49 & 0.27 & 0.07 \\
& RTxAT & -0.0019 & 0.009 & 0.05 \\
& SFxAT & - & - & - \\
& Constant & -4.27 & 2.51 & 0.09 \\
\hline & $R^{2}=0.1517$ & & \\
& $F(5,107)=3.90, p<0.01$ & & & \\
\hline
\end{tabular}

(a)

\begin{tabular}{c|l|lll}
\hline Hypothesis & Antecedent & Coeff. & SE & p \\
\hline H2 & Reaction Time (RT) & 0.33 & 0.13 & 0.01 \\
H9 & SOCIABLE (SF) & -1.08 & 0.45 & 0.01 \\
& Reasoning System (RE) & 4.40 & 1.95 & 0.02 \\
H4 & RTxRE & -0.25 & 0.07 & 0.0006 \\
H5 & SFxRE & -0.50 & 0.23 & 0.035 \\
& Attitude (AT) & 0.11 & 0.34 & 0.73 \\
H7 & RTxAT & -0.006 & 0.012 & 0.59 \\
H8 & SFxAT & 0.038 & 0.046 & 0.40 \\
& Constant & & & \\
\hline & $R^{2}=0.265$ & & \\
& $F(8,104)=4.71, p<0.0001$ & & &
\end{tabular}

(b)

TABLE I: In (a) results of the regression analysis for SOCIABLE (SF) as dependent variable. In (b) the results of the regression analysis for number of Mistakes (M) as dependent variable.

from the robot.

- robot's number_of_feedback: the number of times the participant understands SOCIABLE furnished by the robot and places the token back in its original location.

- participant's number_of_mistakes: the number of times the participant does not perceive the social feedback and places the token in a wrong position.

- participant's reasoning system: according to the two versions of the CRT mentioned in Sec. VI-C.3. Participants scored between 0 and 7 .

- participant's attitude: based on the questionnaire administered to the participant mentioned in Sec. VI-C.3. Participants scored between 3 and 15 .

It is noteworthy that for correct movements we do not have a measure to discern whether SOCIABLE had influence. Thus, in this study we are only interested in evaluating the cases in which the participants grasped a wrong token and realised SOCIABLE.

5) Participants: We recruited 114 participants during a two-day fair. We did not have any withdrawal and all the participants were included in the data analysis. Participants were aged between 18 and 67 (46.1\% of them were female), with $\mathrm{M}=35$ and $\mathrm{SD}=11.77$. The participation in the study was voluntary and no material incentive was offered.

\section{Methodology}

In order to determine the number of samples needed, we based our proposal on i) the assumption that a "minimum of at least five times as many samples as the number of variables is to be analysed" [43] and ii) the report of Fritz and MacKinnon [44] who pointed out that the average sample size for a power of 0.8 is 59 for a percentile bootstrap and 66 for a Sobel test.

To estimate the model depicted in Fig. 4, a regression approach was used, combining dichotomous and continuous variables. We regressed the number of mistakes as a dependent variable, the reaction time and SOCIABLE as independent variables, and lastly SOCIABLE as a mediation variable. We also considered the attitude and the reasoning system as moderating variables, as well as their interactions, using a 5,000-sample bootstrap method with PROCESS version 3 [45].

\section{RESULTS AND DISCUSSION}

Results of our study are reported in Table I. Specifically, Table Ia indicates the results in which SOCIABLE (SF) is the dependent variable, whereas Table Ib indicates the results in which number of Mistakes (M) is the dependent variable.

The last row reported the overall results for the proposed conceptual model. The $R^{2}$ values, which measure how close the data are to the fitted regression model, are aligned with the sample size: $R^{2}$ is equal to 0.1517 when $\mathrm{SF}$ is the dependent variable while $R^{2}$ is equal to 0.265 when $\mathrm{M}$ is the dependent variable. As for both, the overall F-test values are less than the significance level of 0.05 , we can study the individual $\mathrm{p}$-values to find out which of the individual variables are statistically significant. The first column of the Tables reports the hypotheses according to Sec. V, the second column defines the antecedents variables with respect to SF (see Table Ia) and M (see Table Ib), respectively. For each antecedent, the coefficient (Coeff), standard error (SE) and p-value (p) are reported. The magnitude of Coeff for each independent variable signifies the magnitude of the effect that variable has on the dependent variable, and the sign on the coefficient (positive or negative) indicates the direction of the effect. A positive coefficient suggests that as the value of the independent variable increases, the mean of the dependent variable also tends to increase. A negative coefficient suggests that as the independent variable increases, the dependent variable tends to decrease. SE represents the mean distance between the observed value and the regression line (smaller values are better). Finally, the p-value for each term tests the null hypothesis. It is worth mentioning that although in the majority of the HRI studies a p-value of 0.05 has been adopted as a reference of significance, it is common in other fields, such as in consumer behaviour studies, to set it to 0.1 for small samples $(\mathrm{N}=114$, as in our case). Indeed, results between 0.05 and 0.1 are considered a trend and at the limit of significance, and that a further enlarging of samples will validate the results ([46]). Hence, in this study we considered a statistical significance of $90 \%$.

With respect to our research question RQ1 (see Sec. II), that referred to the legibility of the social feedback provided by the robot, 3 of 10 initial hypotheses (H1, H3 and H6) were related to it (see Table Ia), and all of them were 
valid. Results provided evidence that participants devoting more time to placing a token $(\mathrm{H} 1, \mathrm{Coeff}=0.24, \mathrm{p}<0.01)$ were able to understand the feedback (SOCIABLE) provided by the robot (reaction time $>\mu=1.3 \mathrm{~s}$ ). Furthermore, participants with more analytical thinking and that took more time to allocate a token $(\mathrm{H} 3$, Coeff $=0.71, \mathrm{p}<0.05)$ grasped SOCIABLE, namely they made use of it during the interaction. Concerning H6, results showed that participants with a positive attitude toward the robot and higher reaction time did not leverage SOCIABLE (H6, Coeff $=-0.0019, \mathrm{p}<0.05)$. Despite being negative, the value of Coeff is very small, thus we deem this hypothesis needs further investigation, especially in the light of the results we obtained for the single variables AT and RT, which confirmed the opposite. Finally, participants with a higher attitude toward the robot were able to discover SOCIABLE (Coeff $=0.497, \mathrm{p}<0.1)$. As H1 and $\mathrm{H} 3$ were confirmed, we can conclude that SOCIABLE for its intuitiveness and immediacy was understandable as long as the participants were focused or had some degree of motivation and their reaction time was high enough to perceive it.

Regarding RQ2, that referred to the effectiveness of SOCIABLE, 4 of the 10 initial hypotheses (H5, H8, H9, and $\mathrm{H} 10)$ were related to it and three of them, H5, H9 and H10 were valid. Results suggested that participants that were able to leverage SOCIABLE made fewer mistakes (H9, Coeff=$1.08, \mathrm{p}<0.01)$. Additionally, participants with more analytical thinking and who moreover made use of SOCIABLE, made fewer mistakes $(\mathrm{H} 5, \mathrm{Coeff}=-0.5, \mathrm{p}<0.05)$. Despite that, results did not report statistical significance about the ability of participants to detect SOCIABLE moderated by their attitude toward the robot with respect to the number of mistakes $(\mathrm{H} 8$, Coeff $=0.003, \mathrm{p}<0.4)$.

Finally, in order to evaluate the mediator role of SF (H10), we use the Sobel test. The purpose of the test is to evaluate whether the reduction in the effect of the independent variable (RT), after including the mediator in the model (SF), is a significant reduction and therefore whether the mediation effect produced by SF is statistically significant. The test was confirmed, as $\mathrm{H} 1 \mathrm{xH} 9$ returned a value of $\beta=0.243 \mathrm{x}-1.080$ $=-0.262$ that results in 0.150 for Sobel test with $\mathrm{p}<0.05$ ). We can conclude that SOCIABLE caused mediation between the participant's reaction time and number of mistakes.

In addition, three additional hypotheses $(\mathrm{H} 4, \mathrm{H} 2, \mathrm{H} 7)$ were evaluated from the conceptual model presented in Sec. V. With respect to the moderating role of the reasoning system (intuitive vs analytical thinking), we found that participants with more analytical thinking and that devoted more time to locating a token, made fewer mistakes $(\mathrm{H} 4$, Coeff $=-0.258$, $\mathrm{p}<0.001)$. Concerning the moderating role of the degree of involvement of the participants with respect to their reaction time, results did not report statistical significance $(\mathrm{H} 7$, Coeff $=-0.006, \mathrm{p}<0.6)$. Finally, from Table Ib, it can be noted that a higher RT turned out in a higher number of mistakes $(\mathrm{H} 2$, Coeff $=0.33, \mathrm{p}<0.05)$, and higher analytical thinking caused, in turn, the participants to make more mistakes $($ Coeff $=4.40, \mathrm{p}<0.05)$. These last two results are interesting as they are opposed to $\mathrm{H} 4$, meaning that when considered independently, RT and RE had a negative effect on the participants' performance (making more mistakes) whereas the combination of them (RTxRE), in which the RE moderated the relationship between RT and $\mathrm{M}$ led to fewer mistakes.

As a general comment on the results, it seems that attitude (AT) would appear more related to the capability of detecting SOCIABLE, observing that none of the hypotheses including AT are validated in Table Ib. On the contrary, the reasoning system (RE) seems more related to accomplishing the task. Finally, as expected, the participants' reaction time played a key role in the detection of SOCIABLE (see Table Ia). As a matter of fact, playing faster than the time necessary to the robot to furnish SOCIABLE makes it difficult for participants to grasp it. However, from Table Ib a higher reaction time would lead the participants to commit more mistakes. H3 and $\mathrm{H} 4$ provide us an interpretation to disambiguate RT. Indeed, participants with higher reaction time and with more analytical thinking, were capable not only of detecting SOCIABLE but also of committing fewer mistakes.

\section{CONCLUSIONS}

In this work, we aimed to evaluate whether a kind of feedback, we called SOCIABLE, generated by combining verbal and non-verbal backchannel cues and provided promptly, can be perceived by participants interacting with a robot in an entertainment scenario (legibility). Furthermore, we analysed whether and to what extent SOCIABLE can affect their performance (effectiveness). To do so, we built a conceptual model in which participants' attitude and reasoning system functioned as moderating variables, and reaction time and SOCIABLE as antecedents of the number of mistakes. In addition, SOCIABLE was also defined as mediator variable of the relationship between participants' reaction time and their number of mistakes. With the purpose of evaluating the proposed conceptual model, we conducted a two-day study with 114 participants.

Concerning the legibility of SOCIABLE, we provided evidence that the social feedback was comprehended by participants, as when participants took more time in placing a token and they had higher analytical thinking, they were able to perceive SOCIABLE. Moreover, when participants had a positive attitude toward the task, they were able to grasp SOCIABLE.

Regarding the effectiveness of the designed social cue, we showed that the social construct reasoning system, defined as estimators of the participants' cognitive capability, had an impact on their behaviour and thus on their performance. Specifically, results revealed that SOCIABLE when detected by participants that had more analytical thinking, enabled them to make fewer mistakes. Finally, we proved the mediator role of SOCIABLE, namely, the reaction time has an influence on the detection of SOCIABLE which in turn has an influence on the participants' performance.

In summary, we present a pipeline in which a conceptual model is designed to evaluate SOCIABLE's legibility and 
effectiveness, throughout a user study. As future work, we aim to investigate more in-depth the role of attitude, exploring the possibility of measuring it according to a different questionnaire. Furthermore, we plan to extend SOCIABLE, with more complex backchannel cues to evaluate whether we can enhance its effectiveness. Finally, we intend to analyse SOCIABLE's degree of efficiency in a cognitive assistive scenario, whose participants have cognitive impairment.

\section{REFERENCES}

[1] C. Breazeal, K. Dautenhahn, and T. Kanda, "Social Robotics," Springer Handbook of Robotics, vol. 2, pp. 1935-1972, 2016.

[2] H. W. Park, I. Grover, S. Spaulding, L. Gomez et al., "A Model-free Affective Reinforcement Learning Approach to Personalization of an Autonomous Social Robot Companion for Early Literacy Education," in Proceedings of AAAI, vol. 33, no. 1, 2019, pp. 687-694.

[3] C. Clabaugh, K. Mahajan, S. Jain, R. Pakkar et al., "Long-Term Personalization of an In-Home Socially Assistive Robot for Children With Autism Spectrum Disorders," Front. Robot. AI, vol. 6, 2019.

[4] A. Andriella, C. Torras, and G. Alenyà, "Cognitive System Framework for Brain-Training Exercise Based on Human-Robot Interaction," Cognitive Computation, vol. 12, pp. 793-810, 2020.

[5] S. Frennert, H. Eftring, and B. Östlund, "Case Report: Implications of Doing Research on Socially Assistive Robots in Real Homes," Int $J$ of Soc Robotics, vol. 9, no. 3, pp. 401-415, 62017.

[6] J. Fasola and M. J. Matarić, "Using socially assistive human-robot interaction to motivate physical exercise for older adults," Proceedings of the IEEE, vol. 100, no. 8, pp. 2512-2526, 2012.

[7] J. Tolins, K. Liu, M. Neff, M. Walker et al., "A verbal and gestural corpus of story retellings to an expressive embodied virtual character," Proceedings of ICLRE, pp. 3461-3468, 2016.

[8] N. Lazzeri, D. Mazzei, A. Zaraki, and D. De Rossi, "Towards a Believable Social Robot," in Conference on Biomimetic and Biohybrid Systems, 2013, pp. 393-395.

[9] S. M. Fiore, T. J. Wiltshire, E. J. Lobato, F. G. Jentsch et al., "Toward understanding social cues and signals in human-robot interaction: Effects of robot gaze and proxemic behavior," Front. in Psychology, vol. 4, p. 859, 2013

[10] S. Wallkotter, S. Tulli, G. Castellano, A. Paiva et al., "Explainable Agents Through Social Cues: A Review," pp. 1-26, 2020. [Online]. Available: http://arxiv.org/abs/2003.05251

[11] R. E. Petty and J. T. Cacioppo, Communication and Persuasion: Central and Peripheral Routes to Attitude Change. Springer, 2012.

[12] A. Andriella, C. Torras, and G. Alenyà, "Short-Term Human-Robot Interaction Adaptability in Real-World Environments," Int $J$ of Soc Robotics, vol. 12, pp. 639-657, 2019.

[13] S. M. Anzalone, S. Boucenna, S. Ivaldi, and M. Chetouani, "Evaluating the Engagement with Social Robots," Int J of Soc Robotics, vol. 7, no. 4, pp. 465-478, 2015.

[14] J. Kennedy, P. Baxter, and T. Belpaeme, "Nonverbal Immediacy as a Characterisation of Social Behaviour for Human-Robot Interaction," Int J of Soc Robotics, vol. 9, no. 1, pp. 109-128, 2017.

[15] C. Jost, B. L. Pévédic, and D. Duhaut, "Study of the Importance of Adequacy to Robot Verbal and Non Verbal Communication in HumanRobot interaction," in Symposium on Robotics, 2012.

[16] V. Chidambaram, Y. H. Chiang, and B. Mutlu, "Designing persuasive robots: How robots might persuade people using vocal and nonverbal cues," in Proceedings of HRI, 2012, pp. 293-300.

[17] J. Ham, R. H. Cuijpers, and J. J. Cabibihan, "Combining Robotic Persuasive Strategies: The Persuasive Power of a Storytelling Robot that Uses Gazing and Gestures," Int J of Soc Robotics, vol. 7, no. 4, pp. 479-487, 2015.

[18] A. Vinciarelli, M. Pantic, and H. Bourlard, "Social signal processing: Survey of an emerging domain," Image and Vision Computing, vol. 27, no. 12, pp. 1743-1759, 2009.

[19] M. Pantic, R. Cowie, F. D'Errico, D. Heylen et al., "Social Signal Processing: The Research Agenda," in Visual Analysis of Humans, 2011, pp. 511-538.

[20] S. F. Warta, O. B. Newton, J. Song, A. Best et al., "Effects of social cues on social signals in human-robot interaction during a hallway navigation task," Proceedings of the HFES, vol. 2, pp. 1128-1132, 2018.
[21] J. Feine, U. Gnewuch, S. Morana, and A. Maedche, "A Taxonomy of Social Cues for Conversational Agents," International Journal of HCS, vol. 132, pp. 138-161, 2019.

[22] T. J. Wiltshire, E. J. Lobato, D. R. Garcia, S. M. Fiore et al., "Effects of robotic social cues on interpersonal attributions and assessments of robot interaction behaviors," Proceedings of HFES, pp. 801-805, 2015.

[23] P. Jerčić, J. Hagelbäck, and C. Lindley, "Physiological Affect and Performance in a Collaborative Serious Game Between Humans and an Autonomous Robot," in Lecture Notes in Computer Science, vol. 11112, 2018, pp. 127-138.

[24] E. J. Lobato, S. F. Warta, T. J. Wiltshire, and S. M. Fiore, "Varying social cue constellations results in different attributed social signals in a simulated surveillance task," in Proceedings of IFAIRSC, 2015, pp. $61-66$.

[25] T. Turja, I. Aaltonen, S. Taipale, and A. Oksanen, "Robot acceptance model for care (RAM-care): A principled approach to the intention to use care robots," Information \& Management, p. 103220, 2019.

[26] S. E. Beatty and S. M. Smith, "External Search Effort: An Investigation Across Several Product Categories," Journal of Consumer Research, vol. 14 , no. 1 , p. $83,1987$.

[27] H. A. Simon, "A Behavioral Model of Rational Choice," The Quarterly Journal of Economics, vol. 69, no. 1, p. 99, 1955.

[28] I. Ajzen and M. Fishbein, Understanding Attitudes and Predicting Social Behavior., Englewood Cliffs, Ed. Prentice-Hall, 1980.

[29] D. Iacobucci, Marketing Management, Cengage Learning, Ed., 2018.

[30] B. Henri and J. Hartwick, "Measuring user participation, user involvement, and user attitude," MIS Q., vol. 18, no. 1, pp. 59-82, 1994.

[31] J. S. B. Evans, "Intuition and reasoning: A dual-process perspective," Psychological Inquiry, vol. 21, no. 4, pp. 313-326, 2010.

[32] G. Pennycook and D. G. Rand, "Lazy, not biased: Susceptibility to partisan fake news is better explained by lack of reasoning than by motivated reasoning," Cognition, vol. 188, pp. 39-50, 2019.

[33] P. M. Todd, Fast and frugal heuristics for environmentally bounded minds., G. Gigerenzer \& R. Selten, Ed. MIT Press, 2001.

[34] A. W. Kruglanski and G. Gigerenzer, "Intuitive and Deliberate Judgments Are Based on Common Principles," Psychological Review, vol. 118, no. 1, pp. 97-109, 2011.

[35] V. A. Thompson, J. A. Prowse Turner, and G. Pennycook, "Intuition, reason, and metacognition," Cognition, vol. 63, no. 3, pp. 107-140, 2019.

[36] J. S. B. Evans and K. E. Stanovich, "Dual-Process Theories of Higher Cognition: Advancing the Debate," Perspectives on Psychological Science, vol. 8, no. 3, pp. 223-241, 2013.

[37] A. Kalegina, G. Schroeder, A. Allchin, K. Berlin et al., "Characterizing the Design Space of Rendered Robot Faces," Proceedings of HRI, pp. 96-104, 2018.

[38] G. Kanawaty, Introduction to Work Study. International Labour Office, 1992.

[39] B. S. Dhillon, "Methods for performing human reliability and error analysis in health care," International Journal of HCQA, vol. 16, no. 6, pp. 306-317, 2003.

[40] A. Andriella, A. Suárez-Hernández, J. Segovia-Aguas, C. Torras et al., "Natural Teaching of Robot-Assisted Rearranging Exercises for Cognitive Training," in Lecture Notes in Computer Science, vol. 11876. Springer, 2019, pp. 611-621.

[41] M. Heerink, B. Kröse, V. Evers, and B. Wielinga, "Assessing acceptance of assistive social agent technology by older adults: The almere model," Int J of Soc Robotics, vol. 2, no. 4, pp. 361-375, 2010.

[42] W. M. Gervais and A. Norenzayan, "Analytic thinking promotes religious disbelief," Science, vol. 336, no. 6080, pp. 493-496, 42012.

[43] J. F. Hair, W. C. Black, B. J. Babin, and A. RE, Multivariate Data Analysis, New Jersey, Ed. Pearson Prentice Hall, 2010.

[44] M. S. Fritz and D. P. MacKinnon, "Required sample size to detect the mediated effect," Psychological Science, vol. 18, no. 3, pp. 233-239, 2007.

[45] Andrew F. Hayes, Introduction to Mediation, Moderation, and Conditional Process Analysis. The Guilford Press, 2018.

[46] M. Prediger, R. Huertas-Garcia, and J. C. Gázquez-Abad, "How store flyers design affect perceived variety of retailers' assortment," Industrial Management and Data Systems, vol. 119, no. 2, pp. 382396, 2019. 